\title{
Minimal microsatellite shift in microsatellite instability high endometrial cancer: a significant pitfall in diagnostic interpretation
}

\author{
Xinyu $\mathrm{Wu}^{1} \cdot$ Olivia Snir ${ }^{2} \cdot$ Douglas Rottmann $^{1} \cdot$ Serena Wong ${ }^{1} \cdot$ Natalia Buza $^{1} \cdot$ Pei Hui ${ }^{1}$
}

Received: 3 September 2018 / Revised: 31 October 2018 / Accepted: 31 October 2018 / Published online: 15 November 2018

(c) United States \& Canadian Academy of Pathology 2018

\begin{abstract}
Mismatch-repair deficiency testing plays a critical role in the identification of proband in Lynch Syndrome families and triaging patients with high stage or recurrent solid malignancies for check point inhibitor (Pembrolizumab) immunotherapy. We compared microsatellite shift patterns of microsatellite instability PCR analysis at 5 NCI recommended loci between microsatellite instability high endometrial carcinoma $(n=50)$ and microsatellite instability high colorectal cancer $(n=19)$. The endometrial cancer cohort included 45 endometrioid, 1 serous, and 4 clear cell carcinomas. Overall, 52\% (26/50) of microsatellite instability high endometrial cancers showed minimal microsatellite shift (defined as a one to three nucleotide repeat shift at an involved locus) observed at least at one locus. Among microsatellite instability high endometrial cancers with minimal microsatellite shift, the frequencies at each involved locus were D2S123 (21/21, 100\%), D17S250 (10/11, $89 \%)$, D5S346 (11/12, 92\%), BAT25 (9/12, 80\%), and BAT26 (8/21, 45\%). Noticeably, 11 of the 26 cases (42\%) showed only minimal shift. Among microsatellite instability high endometrial cancers with minimal microsatellite shift, $65 \%$ (17/26) had combined MLH1 and PMS2 loss, 8\% (2/26) had combined MSH2 and MSH6 loss, 13\% (3/26) had MSH6 loss and 15\% (4/26) had loss of PMS2 by immunohistochemistry. In contrast, only $16 \%(3 / 19)$ had minimal microsatellite shift seen in colorectal cancer cohort with corresponding loss of MLH1/PMS2, MSH2/MSH6, or MSH6. Overall, 15\% (7/50) of microsatellite instability high endometrial carcinomas showed isolated loss of MSH6 in contrast to 7\% (1/15) seen in microsatellite instability high colorectal carcinomas. In conclusion, microsatellite instability high endometrial carcinomas have a significantly higher frequency of minimal microsatellite shift that coincides with a high percentage of combined loss of MLH1/PMS2. Microsatellite instability high endometrial cancers also have more frequent loss of $M S H-6$. Diagnostically, recognition of minimal microsatellite shift is crucial for accurate interpretation of microsatellite instability PCR data of endometrial carcinoma.
\end{abstract}

\section{Introduction}

Lynch syndrome is an autosomal dominant genetic disorder that predisposes the patient to various types of cancer, especially colorectal and endometrial carcinomas [1-3]. The increased risk of developing these cancers is due to impaired DNA mismatch repair leading to subsequent genetic instability. Microsatellite instability testing and analysis of mismatch-repair protein expression of colorectal

Pei Hui

pei.hui@yale.edu

1 Department of Pathology, Yale University, New Haven, CT, USA

2 Department of Pathology, Oregon Health and Science University, Portland, OR, USA and increasingly endometrial cancers play a critical role in the identification of proband of a Lynch syndrome family [4]. Multiplex polymerase chain reaction-based method is a well-established molecular method to interrogate the microsatellite status, often at the five NCI-recommended loci, with a reported test sensitivity of $93 \%$ in identifying tumors with mismatch repair deficiency [5]. Immunohistochemistry testing evaluates the expression of four mismatch repair proteins (MLH1, PMS2, MSH2, and MSHO), with a reported test sensitivity of over 92\% [6]. Immunohistochemistry has an advantage over microsatellite instability PCR testing in its ability to identify specific mismatch repair gene deficiency, and therefore to guide subsequent comprehensive gene sequencing to identify the causative mutation [7]. Moreover, mismatch repair deficiency testing has recently become a critical companion diagnostic procedure in patients with high stage or recurrent 
solid malignancies for immune check point inhibitor (e.g., Pembrolizumab) therapy $[8,9]$.

In contrast to the well-established colorectal cancer screening algorithm, strategies for cost-effective Lynch syndrome screening of endometrial cancer patients are currently evolving [10] and microsatellite instability high endometrial cancers have been found to prosses distinct pathogenetic differences from colorectal cancers. For example, $B R A F$ mutation is exceedingly rare in endometrial cancers with $M L H 1$ promoter hypermethylation [11] and germline mutations of MSH6 are more often seen in endometrial cancers than colorectal tumors [12]. The aim of this study was to compare the microsatellite shift patterns by microsatellite instability PCR in correlation with mismatch repair protein expression by immunohistochemistry between microsatellite instability high endometrial carcinoma and microsatellite instability high colorectal cancer.

\section{Materials and methods}

\section{Specimens}

The study cohorts included 50 microsatellite instability high endometrial cancers that were identified from 263 endometrial cancers (Table 1) that consecutively underwent combined mismatch repair protein immunohistochemistry and microsatellite instability PCR testing at authors' institution. For comparative analysis, 19 microsatellite instability high colorectal cancers were randomly selected from our departmental archives. Hematoxylin and eosin (H\&E) stained sections and mismatch repair protein immunohistochemical slides were systemically reviewed for each case. Clinicopathological parameters and microsatellite instability

Table 1 Clinicopathological characteristics of MSI-H endometrial cancer cohort

\begin{tabular}{lll}
\hline Age (median age) & $\begin{array}{l}\text { 45 to 80 (62) years } \\
\text { Category }\end{array}$ & No. \\
\hline Histological type & Endometrioid & 45 \\
& Serous & 1 \\
& Clear cell & 4 \\
Histological grade $^{\mathrm{a}}$ & FIGO 1 & 6 \\
& FIGO 2 & 29 \\
& FIGO 3 & 10 \\
Pathological stage $^{\mathrm{b}}$ & FIGO Stage I & 35 \\
& FIGO Stage II & 5 \\
& FIGO Stage III & 6 \\
& FIGO Stage IV & 2 \\
\hline
\end{tabular}

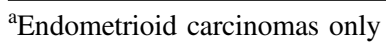

${ }^{\mathrm{b}}$ No staging surgery performed in 3 cases of endometrioid carcinoma
PCR data were revisited and compared between the two cohorts. The study was approved by the institutional review board.

\section{MSI testing by PCR and capillary electrophoresis}

DNA was extracted from tumor tissue and normal tissue of each case according to the pressure treatment and DNA extraction protocol described previously [13]. Microsatellite status was interrogated by microsatellite instability PCR testing at 5 NCI-recommended loci (D2S123, D5S346, D17S250, BAT25, and BAT26). The PCR primers are summarized in Table 1. PCR was performed as follow: denaturation was at $94^{\circ} \mathrm{C}$ for $12 \mathrm{~min}$, followed by 31 cycles of denaturation at $94{ }^{\circ} \mathrm{C}$ for $45 \mathrm{~s}$, annealing at $55^{\circ} \mathrm{C}$ for $45 \mathrm{~s}$, and extension at $72{ }^{\circ} \mathrm{C}$ for $1 \mathrm{~min}$. The last cycle was followed by an extra extension step at $72^{\circ} \mathrm{C}$ for $1 \mathrm{~min}$, then the product was held at $4{ }^{\circ} \mathrm{C}$. PCR products were analyzed by capillary electrophoresis using an ABI 3500 Genetic Analyzer (Applied Biosystems, USA). Status of microsatellite loci was compared between the tumor and its paired nonneoplastic tissue. Microsatellite instability high was defined by the presence of microsatellite instability at two or more loci. microsatellite instability low was defined by the presence of only one unstable locus. Microsatellite stable was defined by the absence of instability at all five loci. Minimal microsatellite shift was defined by shift of 1 to 3 microsatellite nucleotide repeats at an involved locus (Fig. 2a) and major shift was defined by more than 3 microsatellite repeat shifts (Fig. 2b).

\section{MMR gene expression by immunohistochemistry analysis}

Mismatch repair protein immunohistochemistry was performed on 4- $\mu \mathrm{m}$ sections of formalin-fixed, paraffinembedded tumor tissue block that were selected after reviewing $\mathrm{HE}$ stained sections to ensure tissue adequacy in each case. After incubation at $60^{\circ} \mathrm{C}$ for $2 \mathrm{~h}$, the tissue sections were deparaffinized in xylene twice, followed by 5 min each in $99.5 \%$ and $95 \%$ ethanol and $5 \mathrm{~min}$ in distilled water. Tissue sections were then stained for four mismatch repair protein antibodies including $M L H 1, P M S 2, M S H 2$, and MSH6 (Ventana, prediluted) with an incubation time of 60 min. DAKO EnVision + kit (DAKO, Carpinteria, CA) was used for visualization. Positive and negative controls (normal colonic mucosa) were included. Complete lack of nuclear staining of invasive carcinoma cells in the presence of positive staining of inflammatory or stromal cells on the same tissue section was scored as mismatch repair deficient (Fig. 1). 


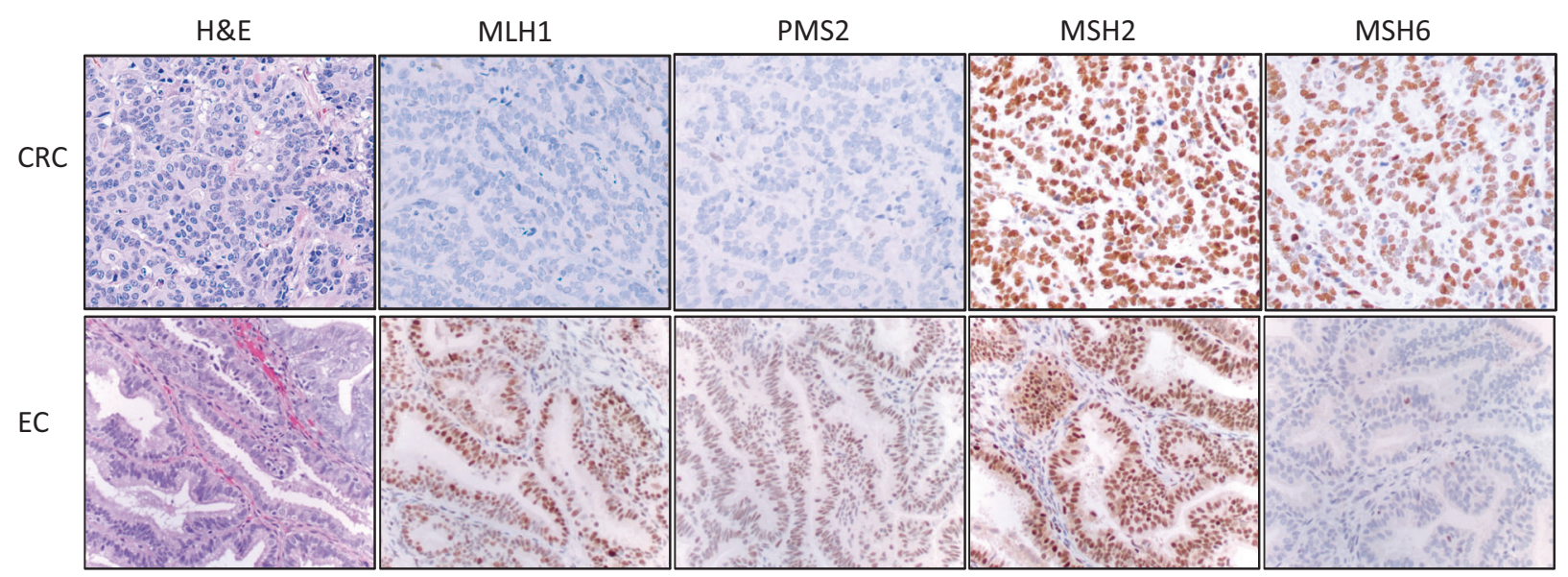

Fig. 1 Mismatch Repair Protein Immunohistochemistry. Top Panel: Combined loss of $M L H 1$ and $P M S 2$ with retained expression of $M S H 2$ and MSH6 in colorectal adenocarcinoma. Lower Panel: Loss of MSH6

\section{Results}

\section{Clinicopathological characteristics of study cohorts}

The microsatellite instability high endometrial cancer study cohort included 45 endometrioid, 1 serous and 4 clear cell carcinomas (Table 1). The age of the patients at diagnosis ranged from 45 to 80 years with a median age of 62 years. Histologic grading of the 45 endometrioid carcinomas included 6 FIGO grade 1, 29 FIGO grade 2 and 10 FIGO grade 3 tumors. Forty-seven patients underwent staging surgery with final pathological stage consisting of 35 stage I (75\%), 5 stage II (11\%), 6 stage III (13\%), and 2 stage IV (4\%) tumors.

The microsatellite instability high colorectal cancer cohort included 19 cases (15 males and 4 females). The age of the patients at diagnosis ranged from 43 to 87 years with a median age of 70 years. All patients had pathologic stage III disease at the time of surgery.

\section{High frequency of minimal microsatellite shift in microsatellite instability high endometrial cancer}

Among microsatellite instability high endometrial cancer cases, microsatellite instability was present at 2 or 3 loci in $66 \%$ (33 of 50 cases) and 4 or 5 loci in 34\% (17 of 50 cases) of cases (Table 2). Microsatellite instability high colorectal cancers showed comparable microsatellite instability with $79 \%$ of cases (15 of 19) involving 2 or 3 microsatellite loci and $21 \%$ of cases (4 of 19) involving 4 or 5 microsatellite loci.

Although both minimal and major microsatellite shifts (Fig. 2a) were observed in the two study cohorts, microsatellite instability high endometrial carcinoma showed a higher frequency of minimal shift involving at least one of and retained expression of $M L H 1, P M S 2$, and $M S H 2$ in a FIGO grade 1 endometrial endometrioid adenocarcinoma

the five loci $(52 \%, 26$ of 50 cases), in contrast to only $16 \%$ of microsatellite instability high colorectal cancers ( 3 of 19 cases) demonstrating minimal microsatellite shift (Table 2).

Among the 26 microsatellite instability high endometrial cancers with minimal microsatellite shift, the minimal shift involved one locus in 6 cases, 2 loci in 10 cases, 3 loci in 8 cases, 4 loci in 2 cases, and 5 loci in none (Table 3). Eleven of these cases $(42 \%, 11 / 26)$ demonstrated only minimal shift at all unstable loci. There were significant variations among the five microsatellite loci involved by minimal shift (Table 3). The frequency of minimal shift at a microsatellite locus that became unstable were $100 \%$ at D2S123 (21/21 cases, Fig. 3), $91 \%$ at $D 17 S 250$ (10/11 cases), $92 \%$ at D5S346 (11/12 cases), $75 \%$ at BAT25 (9/12 cases), and $38 \%$ at $B A T 26$ (8/21 cases).

\section{Combined loss of MLH1 and PMS2 coincides with minimal microsatellite shift in MSI-H endometrial cancer}

Most endometrial carcinoma cases (48 of 50) and colorectal cancer cases (15 of 19) had mismatch repair protein immunohistochemistry performed on the same tumor block analyzed by microsatellite instability PCR. As expected (Table 2), the most common abnormal immunohistochemistry result was the combined loss of $M L H 1$ and $P M S 2$ proteins in both endometrial cancer $(62.5 \%)$ and colorectal cancer (53\%) (31). Five endometrial cancers (10\%) and 4 colorectal cancers (27\%) had combined loss of $M S H 2$ and MSH6. Six endometrial cancers (13\%) and 2 colorectal cancers (13\%) lost PMS2 expression alone and 7 endometrial cancers $(15 \%)$ and 1 colorectal cancer $(7 \%)$ had MSH6 loss alone (Table 2, Fig. 1). Among microsatellite instability high endometrial cancers with minimal microsatellite shift, 65\% (17/26) showed combined MLH1 and 
Table 2 Summary of microsatellite instability PCR testing and mismatch repair protein immunohistochemistry

\begin{tabular}{|c|c|c|c|c|}
\hline & & & $\begin{array}{l}\text { Endometrial cancers } \\
(N=50)\end{array}$ & $\begin{array}{l}\text { Colorectal } \\
\text { cancers } \\
(N=19)\end{array}$ \\
\hline \multirow[t]{4}{*}{ MSI } & \multirow[t]{2}{*}{ No. Loci involved } & 2 to 3 Unstable Loci & $33(66 \%)$ & $15(79 \%)$ \\
\hline & & 4 to 5 Unstable Loci & $17(34 \%)$ & $4(21 \%)$ \\
\hline & \multirow[t]{2}{*}{ Shift pattern } & Minimal Shift & $26(52 \%)$ & $3(16 \%)$ \\
\hline & & Major Shift & $24(48 \%)$ & $16(84 \%)$ \\
\hline \multirow[t]{9}{*}{ MMR } & \multirow[t]{2}{*}{$M L H 1 / P M S 2$ loss } & Overall & $30(63 \%)$ & $8(53 \%)$ \\
\hline & & With minimal shift & $17(65 \%)^{\mathrm{a}}$ & $1(33 \%)^{\mathrm{a}}$ \\
\hline & \multirow[t]{2}{*}{ MSH2/MSH6 loss } & Overall & $5(10 \%)$ & $4(27 \%)$ \\
\hline & & With minimal shift & $2(8 \%)^{\mathrm{a}}$ & $1(33 \%)^{\mathrm{a}}$ \\
\hline & \multirow[t]{2}{*}{$P M S 2$ loss } & Overall & $6(13 \%)$ & $2(13 \%)$ \\
\hline & & With minimal shift & $4(15 \%)^{\mathrm{a}}$ & $0^{\mathrm{a}}$ \\
\hline & \multirow[t]{2}{*}{ MSH6 loss } & Overall & $7(15 \%)$ & $1(7 \%)$ \\
\hline & & With minimal shift & $3(12 \%)^{\mathrm{a}}$ & $1(33 \%)^{\mathrm{a}}$ \\
\hline & Not available & & 2 & 4 \\
\hline
\end{tabular}

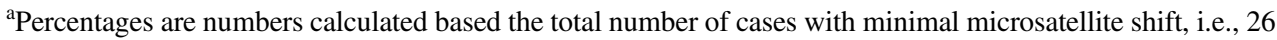
endometrial carcinomas and 3 colorectal carcinomas
PMS2 loss, 8\% (2/26) had combined MSH2 and MSH6 loss, $15 \%(4 / 26)$ and $13 \%(3 / 26)$ showed individual loss of MSH6 or PMS2, respectively (Table 2). Moreover, it is noted that $43 \%$ (3/7 cases) of the MSH6 deficient endometrial carcinomas showed minimal microsatellite shift (Table 2). In contrast, among the 19 microsatellite instability high colorectal cancer cohort, 3 cases had minimal microsatellite shift, corresponding to 1 case of combined loss of MLH1/PMS2, 1 case of combined loss of MSH2/MSH6 and 1 MSH6 loss alone.

\section{Discussion}

The goal of Lynch syndrome screening is to identify involved families, and therefore to provide surveillance for susceptible family members and to prevent additional Lynch syndrome-associated malignancies in the index patient [14]. Beyond Lynch syndrome screening, microsatellite instability testing has recently acquired unprecedented prognostic and predictive value in guiding tumor immunotherapy of patients with advanced microsatellite instability high solid tumors [15-18]. The two most common Lynch syndrome-associated cancers are colon and endometrial cancers with a lifetime risk between $52-82 \%$ and 25-60\%, respectively [2, 19]. Clinicopathologically, Lynch syndrome-associated endometrial cancer is seen in often young patients with a mean age of $48-55$ years [20]. The morphology of Lynch syndrome-associated endometrial cancer is characterized by peritumoral lymphocytes, tumor infiltrating lymphocytes, tumor location in the lower uterine segment, and high histologic grade [21-24]. These gross and histological features may be helpful in guiding patient selection for mismatch repair deficiency testing. Microsatellite instability high endometrial cancers are predominantly of endometrioid histologic subtype of both inherited (Lynch syndrome) and sporadic mismatch repair deficient tumors [24]. Clear cell and serous carcinoma are rarely observed in Lynch syndrome patients [25, 26]. Consistently, $90 \%$ (45 of 50 cases) in our study cohort were endometrioid adenocarcinomas. Clear cell carcinoma was seen in $8 \%$ (4 of 50 cases) and serous carcinoma was seen in one case. Recently, universal screening for Lynch syndrome of all newly diagnosed endometrial cancer patients has been advocated in several clinical recommendations. Our institution has adopted a screening algorithm to reflex all newly diagnosed endometrial cancers for simultaneous microsatellite instability PCR and mismatch repair protein immunohistochemical testing.

In this study, microsatellite instability high endometrial cancers demonstrate a high frequency of minimal microsatellite shift. In a previous comparative study of microsatellite instability profiles of endometrial and colorectal tumors, it was observed, that the size of deletions/insertions differs between colorectal and endometrial carcinomas, in that the latter had shorter alteration or minimal nucleotide shift [27-29]. However, such difference in microsatellite instability nucleotide shift in endometrial carcinoma has not been a common knowledge among practicing molecular pathologists as mismatch repair deficiency testing of endometrial cancer has only become a standard clinical practice very recently. While there are high concordance rates between immunohistochemistry and PCR for colorectal cancer, microsatellite instability PCR testing of 

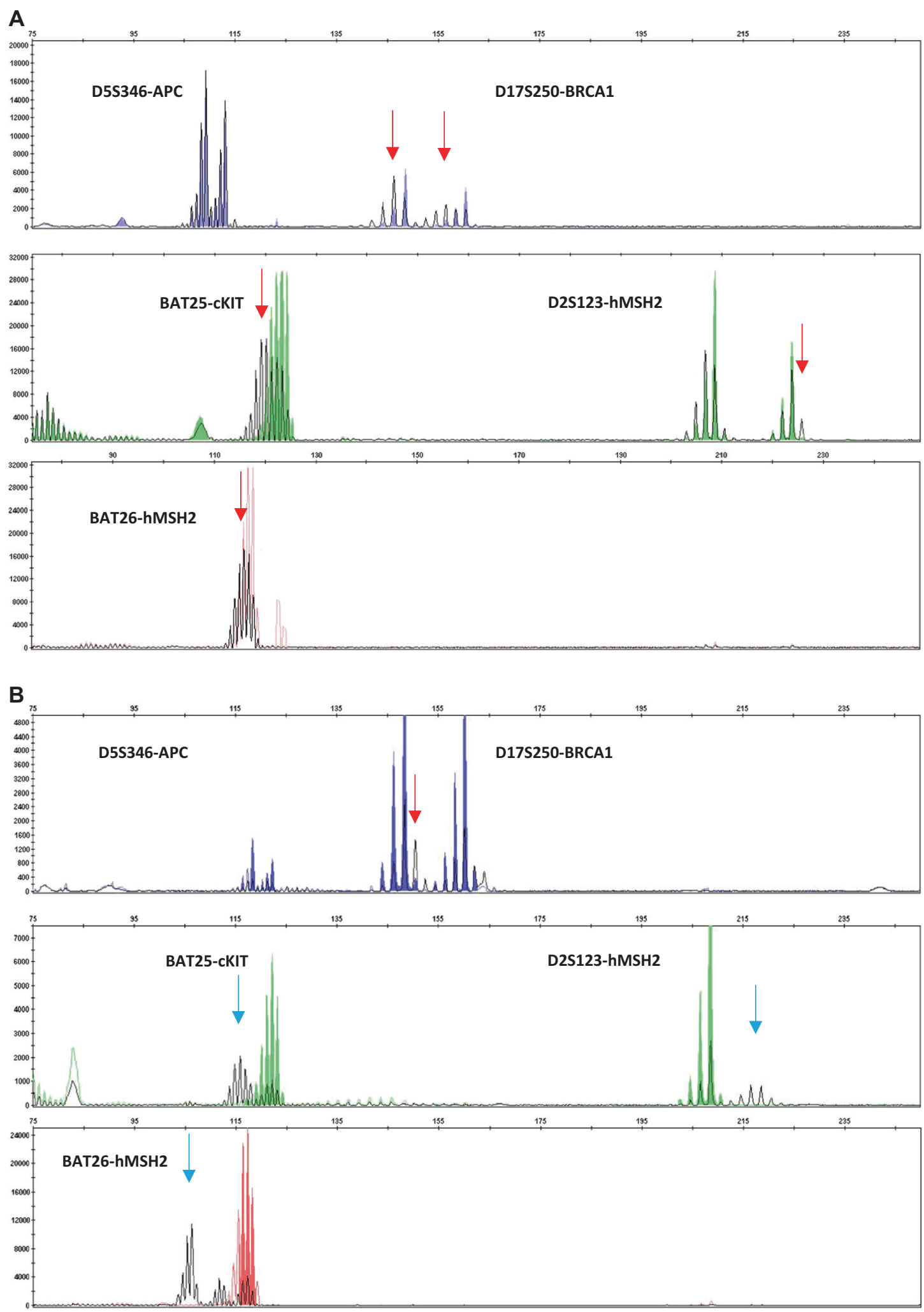

Fig. 2 Microsatellite instability testing by analysis of PCR products amplified at $5 \mathrm{NCI}$ recommended loci. a: Minimal microsatellite shift at four of 5 microsatellite loci (red arrows) in an endometrial endometrioid adenocarcinoma. b: Major microsatellite shifts (blue arrows) and minimal microsatellite shifts (red arrow) in a colorectal carcinoma) 
Table 3 Minimal microsatellite shift patterns at $5 \mathrm{NCI}$ recommended loci in microsatellite instability high endometrial carcinomas $(N=26)$

\begin{tabular}{|c|c|c|c|c|c|c|c|c|}
\hline \multirow[t]{2}{*}{ \# } & \multicolumn{5}{|c|}{ MSI Loci/No. repeat shift } & \multicolumn{3}{|c|}{ Summary of shift pattern } \\
\hline & BAT26 & $D 2 S 123$ & $D 17 S 250$ & D5S346 & BAT25 & $\begin{array}{l}\text { \# Loci minimal } \\
\text { shift }\end{array}$ & $\begin{array}{l}\text { \#Loci major } \\
\text { shift }\end{array}$ & $\begin{array}{l}\text { \% Loci minimal } \\
\text { shift }\end{array}$ \\
\hline 1 & 5 & 0 & 0 & 1 & 0 & 1 & 1 & $50 \%$ \\
\hline 2 & 9 & 3 & 2 & 2 & 2 & 4 & 1 & $80 \%$ \\
\hline 3 & 8 & 1 & 0 & 1 & 0 & 2 & 1 & $67 \%$ \\
\hline 4 & 2 & 2 & 2 & 0 & 0 & 3 & 0 & $100 \%$ \\
\hline 5 & 9 & 5 & 0 & 0 & 1 & 1 & 2 & $33 \%$ \\
\hline 6 & 9 & 3 & 1 & 3 & 4 & 3 & 2 & $60 \%$ \\
\hline 7 & 6 & 3 & 3 & 2 & 3 & 4 & 1 & $80 \%$ \\
\hline 8 & 2 & 0 & 0 & 2 & 0 & 2 & 0 & $100 \%$ \\
\hline 9 & 6 & 2 & 0 & 2 & 3 & 3 & 1 & $80 \%$ \\
\hline 10 & 0 & 2 & 3 & 0 & 0 & 2 & 0 & $100 \%$ \\
\hline 11 & 3 & 0 & 0 & 0 & 2 & 2 & 0 & $100 \%$ \\
\hline 12 & 0 & 1 & 0 & 5 & 0 & 1 & 1 & $50 \%$ \\
\hline 13 & 0 & 2 & 3 & 0 & 0 & 2 & 0 & $100 \%$ \\
\hline 14 & 6 & 2 & 2 & 0 & 2 & 3 & 1 & $75 \%$ \\
\hline 15 & 12 & 2 & 0 & 0 & 0 & 1 & 1 & $50 \%$ \\
\hline 16 & 0 & 2 & 2 & 0 & 0 & 2 & 2 & $100 \%$ \\
\hline 17 & 5 & 2 & 3 & 0 & 2 & 3 & 1 & $75 \%$ \\
\hline 18 & 2 & 2 & 2 & 0 & 0 & 3 & 0 & $100 \%$ \\
\hline 19 & 0 & 2 & 5 & 0 & 0 & 1 & 1 & $50 \%$ \\
\hline 20 & 5 & 2 & 0 & 2 & 5 & 2 & 2 & $50 \%$ \\
\hline 21 & 2 & 2 & 0 & 1 & 0 & 3 & 0 & $100 \%$ \\
\hline 22 & 2 & 1 & 0 & 1 & 0 & 3 & 0 & $100 \%$ \\
\hline 23 & 2 & 0 & 0 & 0 & 2 & 2 & 0 & $100 \%$ \\
\hline 24 & 4 & 0 & 0 & 0 & 2 & 1 & 1 & $50 \%$ \\
\hline 25 & 8 & 2 & 0 & 2 & 8 & 2 & 2 & $50 \%$ \\
\hline 26 & 2 & 2 & 0 & 0 & 0 & 2 & 0 & $100 \%$ \\
\hline
\end{tabular}

endometrial cancer for Lynch syndrome screening has been reported to have a high frequency of false negativity [6, 30], with a particularly low detection sensitivity of MSH6 deficient endometrial cancer [5]. It is possible, that the high frequency of minimal nucleotide shift in endometrial cancers may have contributed to the reported high false negative rate as a result of misinterpretation of minimal shift as stable microsatellite. Corroborating our study, a recent comparative investigation of microsatellite instability PCR profiles between endometrial and colorectal cancer aimed to identify causes of the reported higher false negative rate with microsatellite instability PCR testing [31]. It was found, that $53 \%$ of microsatellite instability high endometrial cancers had a mean absolute shift of 1 to 2 nucleotide repeats (minimal shift) in contrast to only $20 \%$ of microsatellite instability high colorectal cancers showing minimal shift, similar to our findings (minimal repeat shift in $52 \%$ of microsatellite instability high endometrial cancers vs. $16 \%$ in colorectal cancer cases). Taken together, overlooking minimal microsatellite shifting may potentially lead to an incorrect assignment of microsatellite instability high endometrial cancer as microsatellite stable or microsatellite instability low in $42 \%$ (21/50) of our study cases (Table 3). It has been suggested that microsatellite instability PCR testing of tissue samples with less than $30 \%$ of tumor content may not be able to detect the minimal nucleotide shift, further increasing the likelihood of false negativity [31]. Moreover, the standard NCI five microsatellite instability loci may not provide the most sensitive amplicons for microsatellite instability PCR testing. It has been recently advocated that testing additional mononucleotide repeat loci may improve the detection sensitivity by PCR $[32,33]$.

Overall, in our study, $42 \%(11 / 26)$ of cases of endometrial cancer demonstrated only minimal shift and no major shift was observed. It is worth noting, that among microsatellite instability high endometrial cancers, certain loci are more prone to be involved by minimal microsatellite shift (Table 3), particularly D2S123 (21/21, 100\%), D17S250 (10/11, 91\%), and D5S346 (11/12, 92\%). The 
Fig. 3 Microsatellite shift patterns at D2S123 Locus in microsatellite instability high endometrial cancer (orange, $N=50)$ and colorectal cancer (blue, $N=19$ )

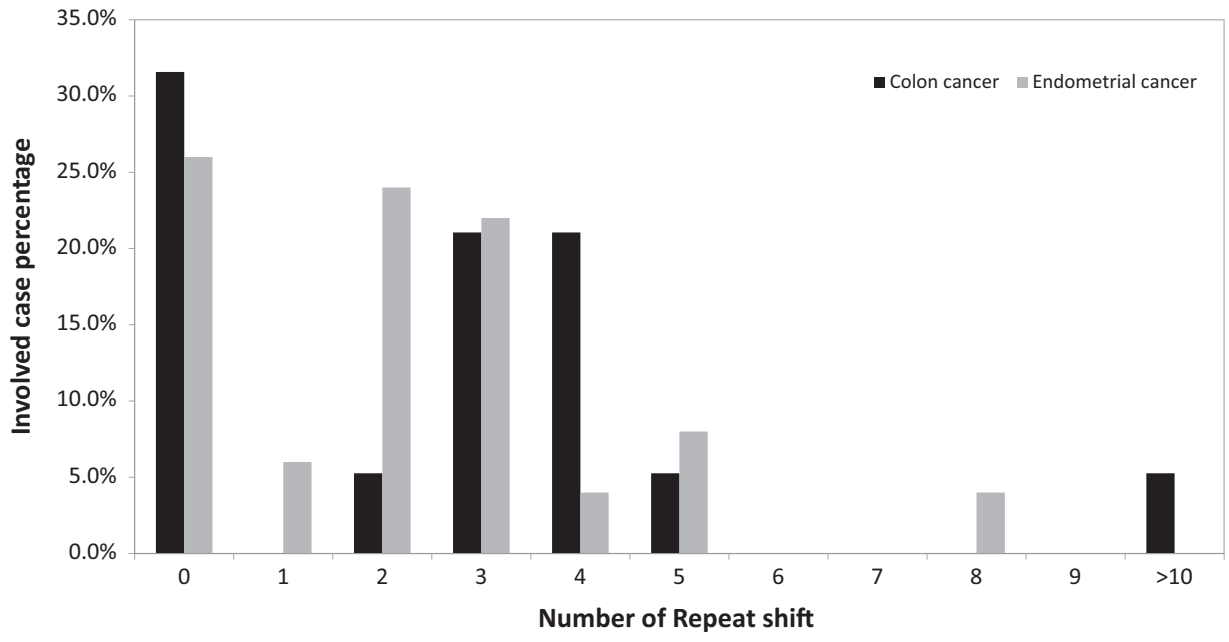

reason for the high frequency of minimal shift in microsatellite instability high endometrial carcinomas is unclear. It has been hypothesized, that the difference in microsatellite instability profiles between microsatellite instability high endometrial and colorectal cancer may be due to more rapid turnover of DNA replication in colorectal cancer, compared to endometrial cancer [31]. Taken together, our data further emphasize, that the high frequency of minimal microsatellite shift is a potential cause of false-negative microsatellite instability PCR result when testing microsatellite instability high endometrial cancers. Awareness of this potential pitfall is essential for accurate interpretation. Careful assessment of small shift peaks and the overall peak patterns, particularly at D2S123, D17S250 and D5S346 loci, are important for accurate interpretation of microsatellite instability PCR data in endometrial carcinoma.

Unlike colorectal cancer, female MSH6 mutation carriers have the highest risk for developing endometrial cancer in older patients (61\%), compared to those with MLH1 (25\%) and $\mathrm{MSH} 2$ mutations (49\%) [34]. While hypermethylation of $\mathrm{MLH} 1$ promoter is common in sporadic endometrial cancer [35] similar to microsatellite instability high colorectal cancers, $B R A F$ V600E mutation is very uncommon in sporadic endometrial carcinomas with $M L H 1$ promoter hypermethylation [11]. Corroborating the literature, our study has confirmed that microsatellite instability high endometrial carcinomas have more frequent minimal microsatellite shift, further emphasizing the genetic differences between microsatellite instability high endometrial carcinoma and microsatellite instability high colorectal carcinomas. It should be noted that such frequent minimal microsatellite shift observed in endometrial cancer has not been reported in other types of microsatellite instability high malignancies. Nevertheless, molecular pathologists should pay close attention to the subtle microsatellite shifting and interpret them as unstable microsatellite loci when testing all tumor types.
The United States Multi-Society Task Force has published a consensus statement regarding screening, genetic evaluation and management for Lynch syndrome-associated colorectal cancers [36]. However, strategies for costeffective screening and subsequent genetic evaluation of endometrial cancer patients are still evolving [10] with varying approaches of integrating mismatch repair protein immunohistochemistry and microsatellite instability PCR testing into the screening algorithms. Mismatch repair protein immunohistochemistry is preferred at some institutions as a primary screening method, supplemented by microsatellite instability PCR testing [37]. The advantages of mismatch repair protein immunohistochemistry include gene specific recognition when abnormal loss of expression of mismatch repair genes is observed, and the interpretation is familiar to pathologists. However, it has been increasingly recognized that interpretation of mismatch repair protein immunohistochemistry of endometrial carcinoma can be problematic. Firstly, similar to colorectal cancers, weak or equivocal staining patterns of mismatch repair protein immunohistochemistry due to technical or tissue fixation issues can lead to significant interobserver variability [38]. Second, not infrequently the foci of mismatch repair deficient invasive carcinoma are closely admixed with atypical endometrial hyperplasia, that may retain normal expression of mismatch repair genes, leading to false negative interpretation. Thirdly, in rare cases, missense mutations of one of the mismatch repair genes may result in a nonfunctional protein but normal immunostaining pattern, leading to false negativity [39]. Lastly, while non-neoplastic cells express nuclear staining of mismatch repair proteins, abnormal cytoplasmic staining of tumor cells may be misinterpreted as normal expression [40].

Microsatellite instability PCR is a sensitive method for the detection of mismatch repair deficient tumors. The limitations of molecular microsatellite instability testing include the requirement of paired normal tissue for testing 
and inability to specify which mismatch repair gene is involved when a tumor has mismatch repair deficiency. Recent studies indicate a high concordance rate between mismatch repair protein immunohistochemistry and microsatellite instability PCR in the workup of endometrial cancer [41]. It is important to recognize that both mismatch repair protein immunohistochemistry and microsatellite instability PCR share similar testing sensitivity of 91 to 93\% and both miss 7-9\% of mismatch repair deficient/ Lynch syndrome-related cancers [10, 37]. Our study has also confirmed a higher frequency of MSH6 loss in microsatellite instability high endometrial carcinomas. Loss of MSH6 frequently leads to minimal microsatellite instability in our study (43\%, 3/7 cases), that may be erroneously misinterpreted as either microsatellite instability low or microsatellite stable tumor. While minimal microsatellite shift may be overlooked and misinterpreted as a false negative PCR result, the limitations of immunohistochemistry interpretation of MSH6 must also be taken into consideration. MSH6 expression level may diminish after chemotherapy or radiation treatment in microsatellite stable tumors, leading to false positive results [39, 42]. Therefore, complimentary co-testing by microsatellite instability PCR and mismatch repair protein immunohistochemistry likely offers the most sensitive and specific approach to capture mismatch repair deficient endometrial carcinomas.

In conclusion, there are fundamental pathogenetic differences between microsatellite instability high endometrial carcinomas and microsatellite instability high colorectal cancers. Compared to colorectal cancers, microsatellite instability high endometrial carcinomas incur a higher frequency of minimal microsatellite shift that coincides with a higher percentage of combined loss of MLH1/PMS2. Microsatellite instability high endometrial cancers also have more frequent loss of MSH6. Diagnostically, recognition of minimal microsatellite shift is crucial for accurate interpretation of microsatellite instability PCR data for Lynch syndrome screening and guiding check point inhibitor immunotherapy for patients with high stage or recurrent endometrial cancers.

\section{Compliance with ethical standards}

Conflict of interest The authors declare that they have no conflict of interest.

\section{References}

1. Scott RJ, McPhillips M, Meldrum CJ, et al. Hereditary nonpolyposis colorectal cancer in 95 families: differences and similarities between mutation-positive and mutation-negative kindreds. Am J Hum Genet. 2001;68:118-27.
2. Stoffel E, Mukherjee B, Raymond VM, et al. Calculation of risk of colorectal and endometrial cancer among patients with Lynch syndrome. Gastroenterology. 2009;137:1621-27.

3. Lynch HT, Smyrk T. Hereditary nonpolyposis colorectal cancer (Lynch syndrome). An updated review. Cancer. 1996;78:1149-67.

4. Shia J. Immunohistochemistry versus microsatellite instability testing for screening colorectal cancer patients at risk for hereditary nonpolyposis colorectal cancer syndrome. Part I. The utility of immunohistochemistry. J Mol Diagn. 2008;10:293-300.

5. Palomaki GE, McClain MR, Melillo S, Hampel HL, Thibodeau SN. EGAPP supplementary evidence review: DNA testing strategies aimed at reducing morbidity and mortality from Lynch syndrome. Genet Med. 2009;11:42-65.

6. Hampel H, Frankel W, Panescu J, et al. Screening for Lynch syndrome (hereditary nonpolyposis colorectal cancer) among endometrial cancer patients. Cancer Res. 2006;66:7810-17.

7. de Leeuw WJ, Dierssen J, Vasen HF, et al. Prediction of a mismatch repair gene defect by microsatellite instability and immunohistochemical analysis in endometrial tumours from HNPCC patients. J Pathol. 2000;192:328-35.

8. Makker V, Green AK, Wenham RM, Mutch D, Davidson B, Miller DS. New therapies for advanced, recurrent, and metastatic endometrial cancers. Gynecol Oncol Res Pract. 2017;4:19.

9. Yu Y. Molecular classification and precision therapy of cancer: immune checkpoint inhibitors. Front Med. 2018;12:229-35.

10. Buza N, Ziai J, Hui P. Mismatch repair deficiency testing in clinical practice. Expert Rev Mol Diagn. 2016;16:591-604.

11. Metcalf AM, Spurdle AB. Endometrial tumour BRAF mutations and MLH1 promoter methylation as predictors of germline mismatch repair gene mutation status: a literature review. Fam Cancer. 2014;13:1-12.

12. Schweizer P, Moisio AL, Kuismanen SA, et al. Lack of MSH2 and MSH6 characterizes endometrial but not colon carcinomas in hereditary nonpolyposis colorectal cancer. Cancer Res. 2001;61:2813-15.

13. Chung JY, Yi JM, Xie R, et al. A pressure cooking-based DNA extraction from archival formalin-fixed, paraffin-embedded tissue. Anal Biochem. 2012;425:128-34.

14. Lynch HT, Boland CR, Gong G, et al. Phenotypic and genotypic heterogeneity in the Lynch syndrome: diagnostic, surveillance and management implications. Eur J Hum Genet. 2006;14:390-402.

15. Ribic CM, Sargent DJ, Moore MJ, et al. Tumor microsatelliteinstability status as a predictor of benefit from fluorouracil-based adjuvant chemotherapy for colon cancer. $N$ Engl J Med. 2003;349:247-57.

16. Guastadisegni C, Colafranceschi M, Ottini L, Dogliotti E. Microsatellite instability as a marker of prognosis and response to therapy: a meta-analysis of colorectal cancer survival data. Eur J Cancer. 2010;46:2788-98.

17. Hutchins G, Southward K, Handley K, et al. Value of mismatch repair, KRAS, and BRAF mutations in predicting recurrence and benefits from chemotherapy in colorectal cancer. J Clin Oncol. 2011;29:1261-70.

18. Arend RC, Jones BA, Martinez A, Goodfellow P. Endometrial cancer: molecular markers and management of advanced stage disease. Gynecol Oncol. 2018;150:569-80.

19. Egoavil C, Alenda C, Castillejo A, et al. Prevalence of Lynch syndrome among patients with newly diagnosed endometrial cancers. PLoS One. 2013;8:e79737.

20. Hampel H, Frankel WL, Martin E, et al. Screening for the Lynch syndrome (hereditary nonpolyposis colorectal cancer). N Engl J Med. 2005;352:1851-60.

21. Garg K, Leitao MM Jr., Kauff ND, et al. Selection of endometrial carcinomas for DNA mismatch repair protein immunohistochemistry using patient age and tumor morphology enhances 
detection of mismatch repair abnormalities. Am J Surg Pathol. 2009;33:925-33.

22. Westin SN, Lacour RA, Urbauer DL, et al. Carcinoma of the lower uterine segment: a newly described association with Lynch syndrome. J Clin Oncol. 2008;26:5965-71.

23. Walsh MD, Cummings MC, Buchanan DD, et al. Molecular, pathologic, and clinical features of early-onset endometrial cancer: identifying presumptive Lynch syndrome patients. Clin Cancer Res. 2008;14:1692-700.

24. Shia J, Black D, Hummer AJ, Boyd J, Soslow RA. Routinely assessed morphological features correlate with microsatellite instability status in endometrial cancer. Hum Pathol. 2008;39:116-25.

25. Carcangiu ML, Radice P, Casalini P, Bertario L, Merola M, Sala P. Lynch syndrome--related endometrial carcinomas show a high frequency of nonendometrioid types and of high FIGO grade endometrioid types. Int J Surg Pathol. 2010;18:21-26.

26. Mills AM, Liou S, Ford JM, Berek JS, Pai RK, Longacre TA. Lynch syndrome screening should be considered for all patients with newly diagnosed endometrial cancer. Am J Surg Pathol. 2014;38:1501-09.

27. Ferreira AM, Westers $\mathrm{H}, \mathrm{Wu}$ Y, et al. Do microsatellite instability profiles really differ between colorectal and endometrial tumors? Genes Chromosomes Cancer. 2009;48:552-57.

28. Furlan D, Casati B, Cerutti R, et al. Genetic progression in sporadic endometrial and gastrointestinal cancers with high microsatellite instability. J Pathol. 2002;197:603-09.

29. Kuismanen SA, Moisio AL, Schweizer P, et al. Endometrial and colorectal tumors from patients with hereditary nonpolyposis colon cancer display different patterns of microsatellite instability. Am J Pathol. 2002;160:1953-58.

30. Ferguson SE, Aronson M, Pollett A, et al. Performance characteristics of screening strategies for Lynch syndrome in unselected women with newly diagnosed endometrial cancer who have undergone universal germline mutation testing. Cancer. 2014;120:3932-39.

31. Wang Y, Shi C, Eisenberg R, Vnencak-Jones CL. Differences in microsatellite instability profiles between endometrioid and colorectal cancers: a potential cause for false-negative results? J Mol Diagn. 2017;19:57-64.
32. Pagin A, Zerimech F, Leclerc J, et al. Evaluation of a new panel of six mononucleotide repeat markers for the detection of DNA mismatch repair-deficient tumours. $\mathrm{Br} \mathrm{J}$ Cancer. 2013;108:2079-87.

33. Goel A, Nagasaka T, Hamelin R, Boland CR. An optimized pentaplex PCR for detecting DNA mismatch repair-deficient colorectal cancers. PLoS ONE. 2010;5:e9393.

34. Ramsoekh D, Wagner A, van Leerdam ME, et al. Cancer risk in MLH1, MSH2 and MSH6 mutation carriers; different risk profiles may influence clinical management. Hered Cancer Clin Pract. 2009;7:17.

35. Kanaya T, Kyo S, Maida Y, et al. Frequent hypermethylation of MLH1 promoter in normal endometrium of patients with endometrial cancers. Oncogene. 2003;22:2352-60.

36. Giardiello FM, Allen JI, Axilbund JE, et al. Guidelines on genetic evaluation and management of Lynch syndrome: a consensus statement by the US Multi-society Task Force on colorectal cancer. Am J Gastroenterol. 2014;109:1159-79.

37. Tafe LJ, Riggs ER, Tsongalis GJ. Lynch syndrome presenting as endometrial cancer. Clin Chem. 2014;60:111-21.

38. Klarskov L, Ladelund $\mathrm{S}$, Holck $\mathrm{S}$, et al. Interobserver variability in the evaluation of mismatch repair protein immunostaining. Hum Pathol. 2010;41:1387-96.

39. Wahlberg SS, Schmeits J, Thomas G, et al. Evaluation of microsatellite instability and immunohistochemistry for the prediction of germ-line MSH2 and MLH1 mutations in hereditary nonpolyposis colon cancer families. Cancer Res. 2002;62:3485-92.

40. Sekine S, Ogawa R, Saito S, et al. Cytoplasmic MSH2 immunoreactivity in a patient with Lynch syndrome with an EPCAMMSH2 fusion. Histopathology. 2017;70:664-9.

41. McConechy MK, Talhouk A, Li-Chang HH, et al. Detection of DNA mismatch repair (MMR) deficiencies by immunohistochemistry can effectively diagnose the microsatellite instability (MSI) phenotype in endometrial carcinomas. Gynecol Oncol. 2015;137:306-10.

42. Bao F, Panarelli NC, Rennert H, Sherr DL, Yantiss RK. Neoadjuvant therapy induces loss of MSH6 expression in colorectal carcinoma. Am J Surg Pathol. 2010;34:1798-804. 